\title{
Neurophysiological evaluation of associated demyelinating peripheral neuropathy and multiple sclerosis: a case report
}

\author{
KR MILLS, NMF MURRAY \\ From the Department of Clinical Neurophysiology, The National Hospital for Nervous Diseases, London, UK
}

SUMmary A case of combined multiple sclerosis and demyelinating neuropathy is presented. Percutaneous electrical stimulation of the cortex and spinal cord has shown that pyramidal tract conduction time was prolonged and conduction velocity in the cord was $4 \mathrm{~m} / \mathrm{s}$. Motor conduction velocity in proximal segments of peripheral nerves was slowed to the same extent as in distal segments.

The coincidence of multiple sclerosis and demyelinating peripheral neuropathy in the same subject is a rare but well recognised phenomenon. ${ }^{1-3}$ In this situation, assessment of the relative importance of peripheral and central lesions affecting the same pathway can be difficult.

The technique of percutaneous stimulation of the brain and spinal cord ${ }^{45}$ permits, for the first time, direct measurement of corticospinal tract conduction in the conscious subject. Anodal shocks from a low output impedance stimulator will excite the motor cortex and evoke muscle action potentials (MAPs) in contralateral muscles; the stimulus voltage required is reduced by partial voluntary contraction of the muscle. Cathodal shocks similarly delivered to the spinal cord will evoke MAPs in ipsilateral muscles without the need for voluntary contraction. Central conduction has been studied by this method in mutiple sclerosis and Parkinson's disease. ${ }^{6-9} \mathrm{We}$ have applied the technique to study pyramidal tract and proximal peripheral nerve conduction in a patient with evidence of both central and peripheral demyelination.

\section{Case report}

A 51-year-old man with no family history of neurological disease developed blurring of vision in both eyes in 1962 . This resolved spontaneously after a month, but a similar episode lasting 2 months occurred 5 years later. In 1968, he

Address for reprint requests: Dr NMF Murray, The National Hospital for Nervous Diseases, Queen Sq. London WCIN 3BG, UK.

Received 25 January 1985 and in revised form 7 June 1985. Accepted 12 June 1985 developed peripheral paraesthesiae and weakness of arms and legs increasing over 5 months when examination revealed distal weakness, reduced sensation in a glove and stocking distribution, loss of deep tendon reflexes and flexor ce plantar responses. Sensory nerve action potentials (SAPS were absent and there was marked slowing of motor nere conduction in arm and leg nerves (median $16 \mathrm{~m} / \mathrm{s}$, later popliteal $26 \mathrm{~m} / \mathrm{s}$ ). The CSF protein was $145 \mathrm{mg} / 100 \mathrm{ml}$ and 음 a sural nerve biopsy specimen showed changes compatible with a demyelinating neuropathy. Symptoms improved ove 3 the following 3 months. In 1970 he developed weakness \& both legs, numbness below the waist, constipation, mict迎 $\stackrel{\circ}{\supset}$ rition difficulty and impotence. On examination there was $\overrightarrow{8} \cdot \overrightarrow{0}$ moderate paraparesis with brisk knee jerks, extensor planta responses and a sensory level at D11. Visual evoked potentials (VEPs) were delayed bilaterally, a myelogram was nor$\mathrm{mal}$ and nerve conduction studies were similar to those of 1968. The diagnosis of multiple sclerosis as well as demyelinating peripheral neuropathy was made on clinical grounds and there was a striking improvement with a course of ACTH injections. Prednisolone therapy was commenced and has continued since then. Since 1970 there have been further episodes, approximately yearly, of limb weakness 3 and sensory impairment resulting in progressive neurological $\bar{T}$ deterioration. Oligoclonal bands were not sought in the CSF in 1970 but were present in 1980 .

On examination in December 1984, following a further relapse in motor, sensory and bladder function, there was bilateral optic disc pallor, distal weakness and wasting in arms and legs and proximal leg weakness. He was areflexic, with extensor plantar responses, glove and stocking sensory $\delta$ impairment and mild ataxia of the limbs. There was widespread thickening of the peripheral nerves but general exam- $O$ ination was otherwise normal with no pes cavus. The CSF protein was $280 \mathrm{mg} / 100 \mathrm{ml}$ with six white cells. Magnetic $\frac{7}{O}$ resonance imaging of the brain showed lesions consistent with multiple sclerosis plaques in the cortex, periventricular $\mathrm{N}$ region and medulla (Dr I Ormerod). Full blood count, ESR, plasma glucose, serum urea and electrolytes were normal as 
were the serum B12 and blood lead and mercury levels. No monoclonal immunoglobulins were found in the serum.

\section{Electrophysiological investigations}

\section{Nerve conduction studies}

Median, ulnar, radial and sural SAPs were absent. Motor conduction velocities were reduced: Median (concentric needle in abductor pollicis brevis) elbow to wrist $30 \mathrm{~m} / \mathrm{s}$, axilla to elbow $27 \mathrm{~m} / \mathrm{s}$; Ulnar (surface electrode on abductor digiti minimi) elbow to wrist 30 $\mathrm{m} / \mathrm{s}$, axilla to elbow $30 \mathrm{~m} / \mathrm{s}$; Lateral popliteal (surface electrode on extensor digitorum brevis) neck of fibula to ankle $24 \mathrm{~m} / \mathrm{s}$. F-wave latencies were also measured in ulnar and peroneal nerves and $\mathrm{F}$-wave conduction velocities ${ }^{10}$ were similarly reduced (table). There was evidence of chronic partial denervation in abductor pollicis brevis and tibialis anterior.

\section{Evoked potential studies}

VEPs were of prolonged latency for both eyes (P100 latency: right eye $134 \mathrm{~ms}$, left eye $116 \mathrm{~ms}$, upper normal limit $114 \mathrm{~ms}$ ) but were of normal amplitude and waveform. Brainstem auditory evoked potentials were normal. Median SEPs showed delayed cortical responses (N20 latency: right $35 \mathrm{~ms}$, left $31 \mathrm{~ms}$, upper normal limit for arm length $21.4 \mathrm{~ms}$ ) with no recordable brachial plexus or cervical components. The right ulnar nerve was stimulated at the wrist, elbow and axilla. No subcortical SEPs were recordable and the

Table Central and peripheral motor conduction studies cortical $\mathrm{N} \overline{20}$ response was severely delayed $(\mathbf{4 4 . 8} \mathrm{ms}$ with stimuli at the wrist, $37 \cdot 2 \mathrm{~ms}$ at the elbow and $32 \cdot 6$ $\mathrm{ms}$ at the axilla). From this data approximate sensory conduction velocities were calculated as $38 \mathrm{~m} / \mathrm{s}$ in the wrist to elbow segment and $29 \mathrm{~m} / \mathrm{s}$ in the elbow to axilla segment of the ulnar nerve. No lumbar, cervical or cortical SEPs were obtained with stimulation of the posterior tibial nerve at the ankle.

\section{Central motor conduction}

A low output impedance stimulator (D180 Isolated Stimulator, Digitimer Ltd) was used to deliver brief shocks $(0.05$ or $0.1 \mathrm{~ms}$ time constant of delay) of up to $700 \mathrm{~V}$ via surface electrodes over the right ulnar and peroneal nerves, spinal cord and cerebral cortex. The patient gave informed consent to the procedure which had local ethical committee approval. Recordings were made with $\mathrm{Ag} / \mathrm{AgCl}$ disc electrodes and responses were amplified (band pass $20 \mathrm{~Hz}$ to $2 \mathrm{kHz}$ ) and displayed on a conventional EMG machine which was interfaced with a Research Machines $380 \mathrm{Z}$ microcomputer for storage and plotting.

Upper limb recordings were made with electrodes over abductor digiti minimi. Stimuli giving maximal MAPs were applied to the ulnar nerve at the wrist, elbow and axilla, and to the cervical cord (cathode between the 6th and 7th cervical spines, anode $6 \mathrm{~cm}$ lateral). For cortical stimulation the anode was placed over the hand area of the contralateral motor cortex, estimated as a point $7 \mathrm{~cm}$ down the inter-aural line

\begin{tabular}{|c|c|c|c|c|c|c|}
\hline $\begin{array}{l}\text { Stimulus } \\
\text { site }\end{array}$ & Segment & $\begin{array}{l}\text { MAP } a m p \\
(m V)\end{array}$ & $\begin{array}{l}\text { Latency } \\
\text { (ms) }\end{array}$ & $\begin{array}{l}\text { Cond velocity } \\
(\mathrm{m} / \mathrm{s})\end{array}$ & $\begin{array}{l}F W \text { lat } \\
(m s)\end{array}$ & $\begin{array}{l}F W C V \\
(m / s)\end{array}$ \\
\hline \multicolumn{5}{|c|}{ Recording over abductor digiti minimi } & & 30 \\
\hline Elbow & $A x-E$ & 3.6 & $13 \cdot 7$ & $29 \cdot 0$ & $49 \cdot 2$ & 30 \\
\hline Axilla & \multirow{2}{*}{ C6-Ax } & $3 \cdot 6$ & $18 \cdot 9$ & $28 \cdot 8$ & $40 \cdot 6$ & $38 \cdot 6$ \\
\hline C6 & & $2 \cdot 4$ & $27 \cdot 3$ & $38 \cdot 0$ & & \\
\hline Cortex & & $1-1 \cdot 5$ & $37 \cdot 5^{*}$ & & & \\
\hline \multicolumn{7}{|c|}{ Recording over tibialis anterior } \\
\hline L4 & L1-L4 & $<0.5 \dagger$ & $28 \cdot 9$ & $26 \cdot 7$ & 59 & 27 \\
\hline L1 & D1-L1 & $<0.5 \dagger$ & $35 \cdot 9$ & $11 \cdot 5$ & & \\
\hline D1 & \multirow{2}{*}{ C6-D1 } & $<0.5 \dagger$ & $123 \cdot 0$ & $4 \cdot 0$ & & \\
\hline C6 & & $<0.5 \dagger$ & $136 \cdot 7$ & $4 \cdot 4 \ddagger$ & & \\
\hline \multicolumn{2}{|l|}{ Cortex } & Absent & & & & \\
\hline
\end{tabular}

W:Wrist. E:Elbow. Ax:Axilla. FH:Fibular head.

FW lat:F-wave latency. FWCV:F-wave conduction velocity.

*The cortex to cord conduction time of $10.2 \mathrm{~ms}$ is prolonged.

Normal value: $4.4 \mathrm{~ms}$ SD 0.75 .

†Responses too dispersed to measure accurately.

$\ddagger$ Normal value: $67 \cdot 4 \mathrm{~m} / \mathrm{s}$ SD $9 \cdot 1 .^{8}$ 
from the vertex and $1 \mathrm{~cm}$ anterior, with the cathode 6 $\mathrm{cm}$ anterior. The subject made a weak voluntary contraction of abductor digiti minimi and the voltage of the shocks was increased until the response latency did not change; no attempt was made to produce responses of maximal amplitude from cortical shocks. Conduction time between the cortex and the cervical cord was abnormally long at $11.2 \mathrm{~ms}$, the mean value ${ }^{7}$ in 15 healthy subjects ( 30 sides) being $4.4 \mathrm{~ms}$, SD 0.75 ms (fig a, table). There was also clear evidence of abnormally slow conduction in the proximal segment of the peripheral nerve: cervical cord to axilla conduction time was $8.9 \mathrm{~ms}$ compared to a mean of $4.1 \mathrm{~ms} \mathrm{SD}$ $0.6 \mathrm{~ms}$ in the same 15 control subjects. MAPs were of reduced amplitude with stimulation at all sites in keeping with a peripheral neuropathy. The conduction velocity in the axilla to elbow and elbow to wrist segments of the ulnar nerve was $29 \mathrm{~m} / \mathrm{s}$, virtually identical to the results obtained using a conventional stimulator.

For measurement of central motor conduction to the lower limb, recordings were made with surface

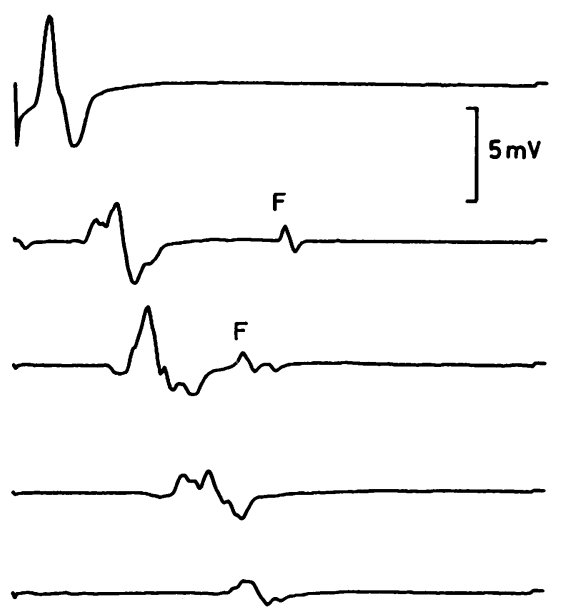

Wrist

Elbow

Axilla

Cord (C6)

Cortex

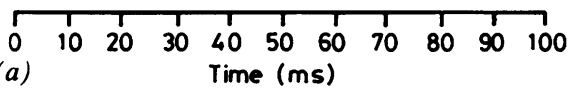

electrodes on tibialis anterior. Stimuli were applied to $\underline{\underline{O}}$ the peroneal nerve at the neck of the fibula and to the $\bar{z}$ cauda equina at the L4 level, with the cathode over the $\stackrel{\mathbb{D}}{\stackrel{D}{ }}$ vertebral column and the anode $6 \mathrm{~cm}$ lateral. The spinal cord was stimulated at lumbar, upper thoracic $\stackrel{\circ}{=}$ and cervical levels; electrodes were aligned vertically 0 with the cathode over the lumbar enlargement of (D12/L1 interspace), D1 and C6 levels and the anode $6 \mathrm{~cm}$ caudal to the cathode. For cortical stimulation, the anode was placed at the vertex and the subject $\vec{z}$ made a weak voluntary contraction of tibialis anterior.

MAPs of onset latency $5.5 \mathrm{~ms}$ and amplitude $5.3 \overrightarrow{0}$ $\mathrm{mV}$ were obtained with peroneal nerve stimulation. 흠 Stimulation over the spine produced small, dispersed $\frac{\bar{\sigma}}{\overline{0}}$ responses of less than $0.5 \mathrm{mV}$ amplitude but with $\overparen{D}$ clearly reproducible onset latencies (fig $b$, table). No responses were obtained with stimuli over the leg area ${ }^{\infty}$ of the motor cortex. There was profound slowing of $\vec{\circ}$ motor conduction over the whole length of the spinal $\overrightarrow{\vec{\omega}}$ cord; the calculated velocity in the C6-D1 segment $\stackrel{\omega}{\sigma}$ was $4.4 \mathrm{~m} / \mathrm{s}$ and in the Dl-L1 segment was $4.0 \mathrm{~m} / \mathrm{s}$.
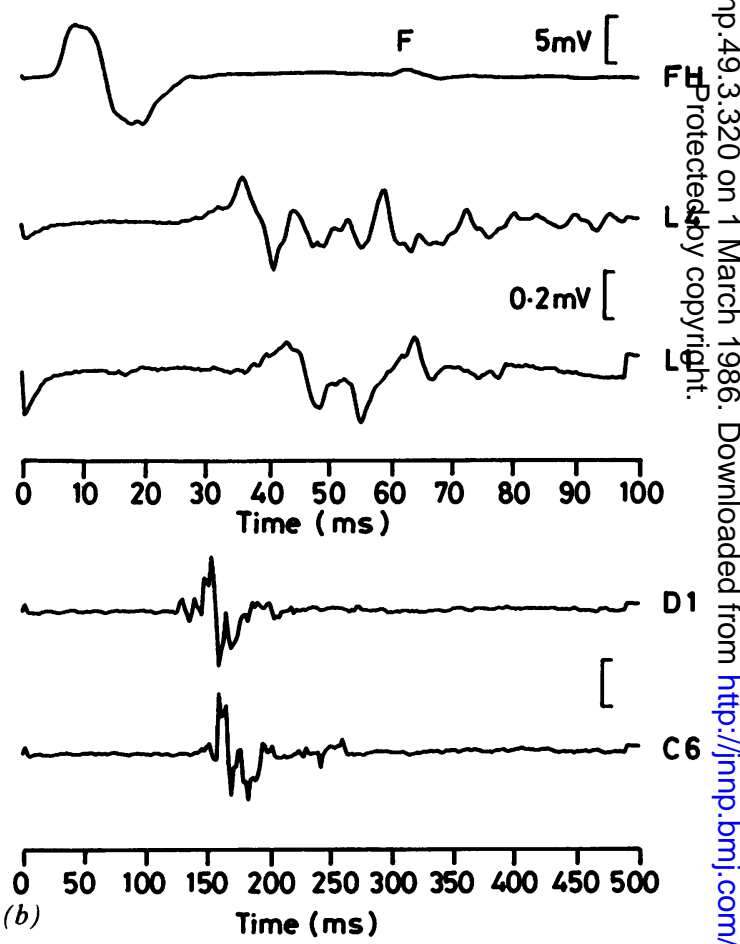

Fig (a) MAPs recorded from right ADM in response to stimulation of the ulnar nerve at the wrist, elbow and axilla, and to stimulation of the spinal cord (C6 level) and the hand area of the left motor cortex. Time and amplitude calibrations are the same for all traces. $F$-waves $(F)$ can be seen in the responses from stimulation at the elbow and axilla. (b) MAPs recorded from right tibialis anterior in response to stimulation of the common peroneal nerve at the fibular head (FH), the cauda equina (L4), and the spinal cord at L1, D1 and C6 vertebral levels. An F wave (F) can be seen in the response from stimulation at the fibular head. The responses from stimulation at points rostral to the fibular head are markedly delayed, dispersed and reduced in amplitude. 
There was severe but less marked slowing of conduction in the proximal peripheral nerve and motor roots: $26.7 \mathrm{~m} / \mathrm{s}$ between $\mathrm{L} 4$ and the fibular head and $11.5 \mathrm{~m} / \mathrm{s}$ between $\mathrm{Ll}$ and $\mathrm{L} 4$.

\section{Discussion}

The new techniques of brain and spinal cord stimulation have allowed direct measurement in this patient of conduction times in the pyramidal tract and in the proximal segment of the peripheral nerves. It has been demonstrated that motor conduction velocity was uniformly reduced over the whole course of the peripheral nerve from spinal cord to muscle and was similar in upper and lower limb nerves. In contrast, slowing in central fibres was patchy; the cortex to cord conduction times were prolonged by a factor of about three, whereas conduction velocity in the spinal motor fibres was about 10 times slower than in healthy subjects. ${ }^{8}$ Similarly, there was a marked asymmetry in the delay of the VEP from the two eyes. These discrepancies would tend to favour a different pathogenesis for the central and peripheral lesions rather than a single diffuse disorder of all myelinated fibres.

The extreme delay in leg muscle responses on stimulating the cervical cord raised the possibility of some indirect or reflex pathway operating, but the finding of shorter latency responses on stimulating the thoracic segments rules this out. Although very slow, a velocity of $4 \mathrm{~m} / \mathrm{s}$ represents a similar degree of slowing to that found in the upper pyramidal tract in some patients with severe multiple sclerosis ${ }^{7}$ where cortex to cord conduction times were prolonged up to $32 \mathrm{~ms}$, the value in healthy controls being $4.4 \mathrm{~ms}$ This preferential involvement of cord fibres also correlates with the clinical picture in which most of the relapses have probably been at spinal level.

In such a case, it may be difficult clinically to distinguish weakness due to neuropathy from that due to pyramidal involvement. Conventional neurophysiological studies such as SEPs may also fail to distinguish between peripheral and central slowing since the marker of the arrival of an ascending volley at the plexus or cord may well be absent and it is then impossible to determine the cause of a delayed cortical response. Thus in this case the central component of the sensory abnormality is uncertain. However, using the ulnar SEP data we can calculate that if central sensory conduction time (cord to cortex) were normal, then conduction in the axilla to cord segment of the nerve would be only $15 \mathrm{~m} / \mathrm{s}$. On the assumption that central sensory and motor conduction were similarly affected then an approximate velocity in the proximal peripheral nerve of $27 \mathrm{~m} / \mathrm{s}$ would be obtained, which is in better agreement with our other findings.

In conclusion, this case illustrates the direct and accurate evaluation of central and proximal peripheral conduction that can be achieved using percutaneous stimulation of the brain and spinal cord.

We thank Prof RW Gilliatt and Dr R Hierons for permission to report the patient under their care and for helpful discussions.

\section{References}

${ }^{1}$ Schoene WC, Carpenter S, Behan PO, Geschwind N. "Onion bulb" formations in the central and peripheral nervous system in association with multiple sclerosis and hypertrophic polyneuropathy. Brain 1977;100: 755-75.

${ }^{2}$ Lassman H, Budka H, Schnaberth G. Inflammatory demyelinating polyradiculitis in a patient with multiple sclerosis. Arch Neurol 1981;38:99-102.

${ }^{3}$ Ro YI, Alexander B, Oh SJ. Multiple sclerosis and hypertrophic demyelinating peripheral neuropathy. Muscle Nerve 1983;6:312-6.

${ }^{4}$ Merton PA, Morton HB, Hill DK, Marsden CD. Scope of a technique for electrical stimulation of human brain, spinal cord, and muscle. Lancet 1982;ii:597-600.

${ }^{5}$ Marsden CD, Merton PA, Morton HB. Percutaneous stimulation of spinal cord and brain: pyramidal tract conduction velocities in man. $J$ Physiol (Lond) 1980;312:5P.

${ }^{6}$ Cowan JMA, Dick JPR, Day BL, Rothwell JC, Thompson PD, Marsden CD. Abnormalities in central motor pathway conduction in multiple sclerosis. Lancet 1984; ii:304-7.

${ }^{7}$ Murray NMF, Mills KR. Pyramidal tract conduction time in normal subjects and multiple sclerosis patients. Muscle Nerve 1984;7:563.

${ }^{8}$ Swash M, Snooks S. Motor conduction velocity in the human spinal cord. J Physiol (Lond) 1985;360:50P.

${ }^{9}$ Beradelli A, Cowan JMA, Day BL, Dick JPR, Marsden CD. Motor cortical excitability in Parkinson's disease. J Physiol (Lond) 1984;353:33P.

${ }^{10}$ Kimura J. Proximal versus distal slowing of motor nerve conduction velocity in the Guillain-Barre syndrome. Ann Neurol 1978;3:344-50. 